\title{
resistencia al fuego de elementos estructurales prefabricados de hormigón armado
}

\author{
MACIEJ ZIOLEK, ingeniero
}

simopsis

839-11

\begin{abstract}
Trata este artículo del comportamiento de estructuras y elementos prefabricados de hormigón armado ante la acción del fuego, con vistas a adoptar los espesores de revestimientos, los materiales, los sistemas, los tipos de juntas e, incluso, las condiciones de explotación de los edificios a construir, que cumplan más adecuadamente con unas determinadas y mínimas propiedades de aislamiento y resistencia.
\end{abstract}

\section{Introduceión}

La amplia aplicación de los prefabricados en la construcción ocasiona el hecho de que, independientemente de la evaluación del edificio con respecto a su estructura, a los materiales, a las condiciones de calorhumedad y acústicas, y de protección contra la acción corrosiva, sea imprescindible tener en cuenta la resistencia al fuego.

La evaluación del edificio desde el punto de vista de la seguridad contra los incendios y la resistencia al fuego debe referirse principalmente a los elementos de construcción que, en forma decisiva, pueden influir sobre la seguridad total del edificio; tanto más cuanto que, en la construcción de prefabricados, existe la tendencia justificada, por razones estructurales, materiales o técnico-económicas, a la aplicación de los elementos con menor revestimiento del acero, generalmente sin enlucidos, con diferentes tipos de juntas y a veces con capas de diferentes materiales de aislamiento.

Por lo tanto hay que tener en cuenta dónde está el límite teórico y práctico del ahorro en la estructuración y realización de los elementos prefabricados, desde el punto de vista de la resistencia al fuego; es éste un criterio muy importante para la evaluación de los edificios prefabricados.

La resistencia al fuego de las estructuras prefabricadas es un problema complejo y depende tanto de las propiedades respectivas de los materiales utilizados, de la forma de su utilización (juntas, sistema de conexión entre los elementos), como también de las condiciones posibles de explotación de los locales.

Basta indicar como ejemplos, que el acero, material incombustible a altas temperaturas, pierde, sin embargo, sus propiedades estructurales; o que las rocas naturales, bajo la acción térmica, sufren descomposición mecánica o química.

En la República Federal Alemana, por ejemplo, se sometieron a investigación forjados de hormigón armado - de un espesor de $12 \mathrm{~cm}$ - y de madera protegidos de la acción de las altas temperaturas, de acuerdo con los requisitos contenidos en la norma DIN 4102 . De las investigaciones realizadas se comprobó que los forjados de madera sufrieron la destrucción al cabo de 1 hora (clase C), mientras que los forjados de hormigón al cabo de 32 y 42 minutos (clase D).

La destrucción de los paneles de hormigón armado fue determinada por las deformaciones permanentes del acero de la armadura originadas por el espesor demasiado pequeño de las capas de hormigón $(1 \mathrm{~cm})$ que protegían las barras. Como principal factor de la destrucción se pueden citar, pues, las deformaciones duraderas del acero de las armaduras bajo la influencia de las altas temperaturas en el caso de un revestimiento de las barras igual a 10 milímetros.

Reafirman este hecho las investigaciones polacas realizadas sobre paneles aligerados de hormigón con acero especial trenzado en forma de cables, en los cuales los recubrimientos del acero son de 10-12 $\mathrm{mm} \mathrm{y}$ los paneles sufrieron la destrucción después de 45 minutos.

La resistencia al fuego del elemento de hormigón armado depende no sólo de la particularidad de los materiales que se forman (siempre que dichos materiales no sean inflamables: como acero y hormigón), sino también de su modo de empleo. 


\section{Definieiones fundamentales}

La resistencia al fuego del elemento depende de la temperatura a la cual está sujeta la estructura, así como del tipo de la intensidad y los cambios de esta temperatura, del tiempo de exposición a la acción de la temperatura, del grado de la dilatación bajo la influencia del calor, del grado de la disminución (en altas temperaturas) de la resistencia de los materiales a los factores mecánicos y del grado de la humedad contenida en las estructuras.

Se llama grado de resistencia al fuego de un elemento, el tiempo durante el cual éste es capaz de desempeñar las funciones para las cuales está previsto; es decir: durante ese tiempo no debe ser destruido ni deformarse de manera excesiva, constituyendo una pantalla contra las llamas y los humos, y no sobrepasar los $150^{\circ} \mathrm{C}$ su temperatura superficial en la cara no expuesta al fuego.

De manera que el criterio principal en las investigaciones y en la determinación de la resistencia al fuego es el correspondiente al tiempo mínimo (dependiente de la clase), al cual puede estar expuesto el elemento dado en las determinadas temperaturas (crecientes en el tiempo) según la curva normativa.

Como se sabe, cada elemento de hormigón armado, independientemente del espesor del revestimiento de la armadura, de la forma y del grosor del elemento, en base a los reglamentos vigentes o en el resultado de las investigaciones realizadas, lo podemos incluir a una clase correspondiente de resistencia al fuego (más de $3,2,5,2,1,5,1,0,75,0,5$ y 0,25 horas) según el país.

\section{La curva normativa «temperatura-tiempo»}

El incendio es un fenómeno complejo que depende del tipo de los materiales inflamables, de la cantidad de estos materiales, del tipo de locales y de las condiciones del medio (temperatura, presión, composición de la atmósfera, dirección del viento, humedad relativa).

En cada incendio, independiente de su curso específico, podemos distinguir los siguientes rasgos esenciales: la temperatura máxima, la cantidad de calor producido (la velocidad de la producción del calor), la velocidad de la combustión; el tiempo (tiempo de la duración del incendio y la velocidad con la cual aumentan las temperaturas).

El fenómeno de incendio podemos dividirlo en tres períodos o fases principales:

1. Fase inicial. Depende de la fuente de encendido y del material (dura de 3 a 50 minutos).

2. $\quad$ Fase de las temperaturas máximas. Es esencial desde el punto de vista constructivo, ya que la estructura se halla en esta fase más expuesta a las deformaciones duraderas, hasta su destrucción (las temperaturas en esta fase oscilan entre $600^{\circ}$ y $1.300^{\circ} \mathrm{C}$ ).

3. Fase de la extinción. En la cual, una vez llegado al equilibrio térmico en la 2. Fase se produce la extinción del incendio, ya que los gases volátiles del material combustible están agotados.

Para obtener las condiciones comparables, los elementos son examinados en los hornos de laboratorio, donde se encuentran sujetos a la acción del fuego según la curva normativa «temperatura-tiempo".

Hasta ahora, como vemos en la figura 1, en los países dados habían sido utilizadas diferentes curvas «temperatura-tiempo», y últimamente todos los países se adaptan a la curva normativa por la ISO. Tam bién la curva determinada para los países miembros del CAME prácticamente se diferencia poco (como vemos en la tabla aneja, en la figura 1, U.R.S.S., Polonia) de la curva de la ISO.

Es característico al principio un considerable aumento del incendio en la 1. Fase (fig. 1), lo que está relacionado con la consideración de las causas frecuentes de los incendios, tales como las explosiones de los materiales fácilmente inflamables.

\section{La carga del fuego}

La carga del fuego es la cantidad de calor que pueden desprender en el curso de un incendio los materiales combustibles comprendidos en el edificio. Este potencial calorífico está expresado, en general,; en megacalorías por metro cuadrado de forjado $\left(\mathrm{Mcal} / \mathrm{m}^{2}\right)$.

Para unificar la determinación de la cantidad del material combustible que corresponde por superficie dada, se utiliza como equivalente la cantidad de madera cuyo calor, promedio de combustión, es igual a $4.400 \mathrm{kcal} / \mathrm{kg}$.

Según las normas inglesas y americanas (Fire Gramding of Building), la carga del fuego equivalente a la intensidad del incendio de 1 hora es igual a $217.000 \mathrm{kcal} / \mathrm{m}^{2}$, es decir, $50 \mathrm{~kg} / \mathrm{m}^{2}$ de madera de un valor térmico promedio de $4.400 \mathrm{kcal} / \mathrm{kg}$. 


\section{Método de las investigaeiones y de las valoraciones de la resistencia al fuego}

Como métodos fundamentales de las investigaciones de la resistencia al fuego de los elementos de la cons. trucción y de las obras podemos mencionar:

las pruebas al fuego en los hornos de laboratorios;

las pruebas al fuego a escala natural;

las pruebas al fuego a escala reducida;

la valoración de la obra y de los elementos según el llamado sistema de puntos.

\section{Las pruebas al fuego en los hornos del laboratorio}

Las investigaciones en los hornos de laboratorio, donde la temperatura crece exactamente según la curva normativa "temperatura-tiempo", con la fluencia constante regulada del combustible y de salida de los productos de la combustión, se lleva a cabo en la mayoría de los países europeos (Gran Bretaña, Francia, U.R.S.S., R.F.A., Italia, Japón, EE.UU., Canadá, etc.).

Las investigaciones se llevan a cabo, con mayor frecuencia, a escala natural. La construcción de los hornos de laboratorio es, en general, similar $y$, dependiendo de las condiciones locales, pueden examinarse en ellos elementos de forjados con las dimensiones de $3,6 \times 4,0 \mathrm{~m}$ (Francia), ó 3,0 ×6,5 m (Italia); es decir: elementos representativos para los empleados en la práctica.

En Brunswick (R.F.A.) hay instalado un horno destinado a investigaciones de elementos de $12 \mathrm{~m}$ longitud, mientras que en los Estados Unidos se utilizan hornos que contemplan la influencia de la fijación de los elementos. En Polonia se están terminando los trabajos de proyecto de los hornos de laboratorio, previstos para la construcción, en el Instituto para la Técnica de la Construcción; la envergadura de los elementos de construcción se prevé hasta $12 \mathrm{~m}$, y la del muro hasta la altura de 3 metros.

\section{Las pruebas al fuego a escala natural}

El desarrollo de la prefabricación en diferentes países, incluida Polonia, con la falta de un horno propio de laboratorio, contribuyó a la necesidad de realizar las pruebas al fuego en las condiciones de polígono. Las pruebas al fuego, a escala natural, no se limitan a la investigación del elemento dado, sino que permiten valorar la influencia de otros factores (ventanas, superficies de las ventanas, balcones, viento). Así, por ejemplo, la sección de control francesa de investigación de incendios, independientemente del horno de laboratorio, había construido un edificio de varias plantas que facilita las investigaciones de los forja-

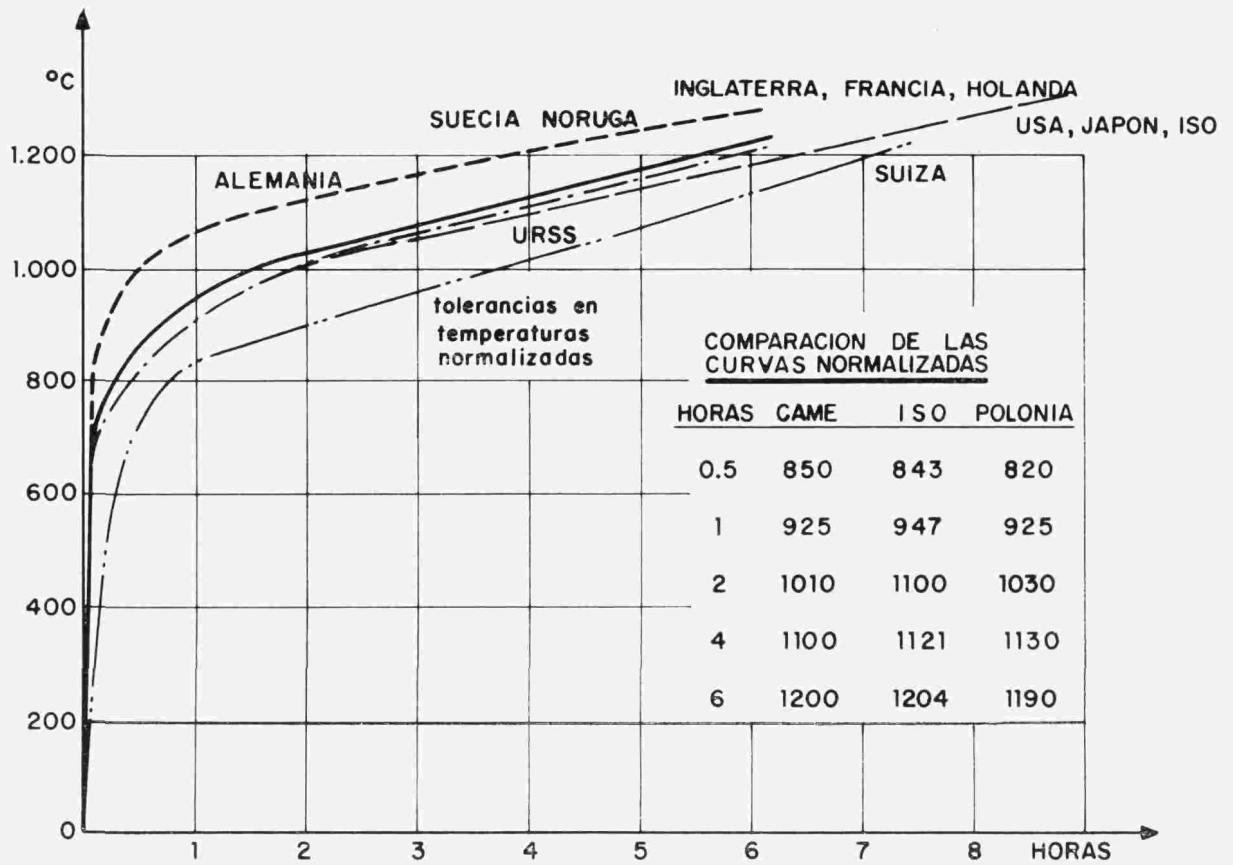



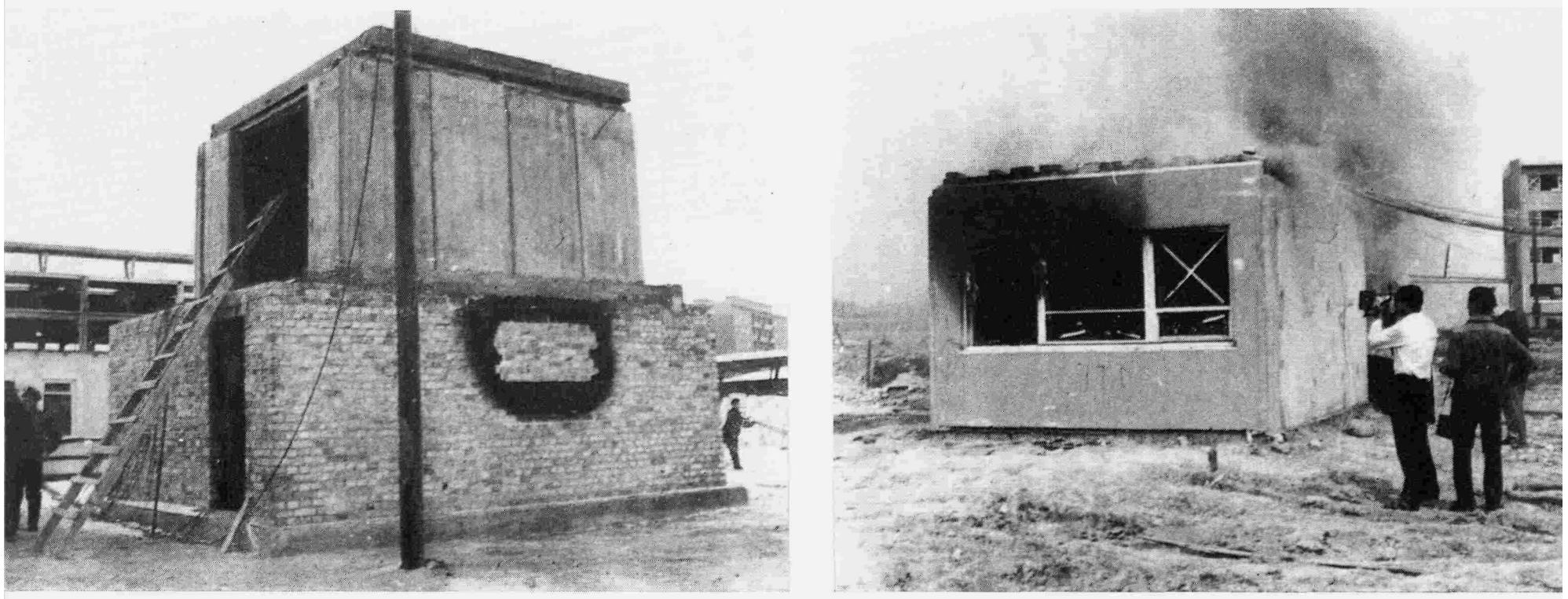

dos, de las paredes y de los pilares, así como las influencias de los balcones, de las ventanas, de la dirección del viento, etc. Tales investigaciones son esenciales y necesarias si tomamos en consideración las tendencias generales al aumento de las aberturas de ventanas o el empleo de diferentes tipos de soluciones estructurales de los nudos prefabricados.

En Polonia, en el Instituto para la Técnica de la Construcción, se han realizado las siguientes pruebas, a escala natural:

de los elementos prefabricados:

los paneles forjados $\mathrm{y}$ de paredes ahuecadas (fig. 2);

de los grandes paneles de forjados y de paredes macizas (fig. 3);

los paneles tipo de cajón nervado y otros.

La medida de las temperaturas en los puntos característicos dados se ha llevado a cabo con ayuda de termopares (fig. 4).
A)

TERMOPARES DE CROMEL - ALUMEL PARA LA MEDIDA DE LAS TEMPERATURAS DE LAS BARRAS DE ARMADURA
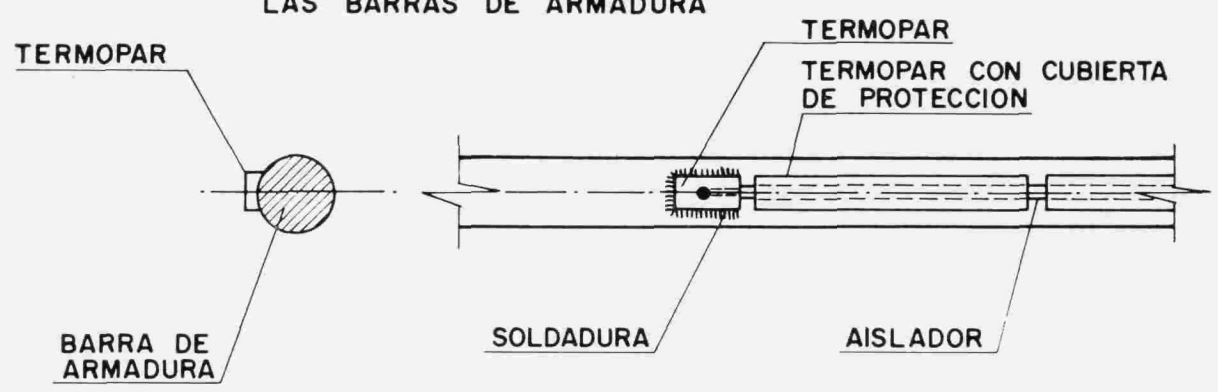

B) TERMOPARES DE CROMEL-ALUMEL PARA LOS ENTREPISOS Y LAS PAREDES EN FORMA DE LAMINA DE TOPE CON $5 \mathrm{~mm}$ TERMINAL DE CROMEL - ALUMEL
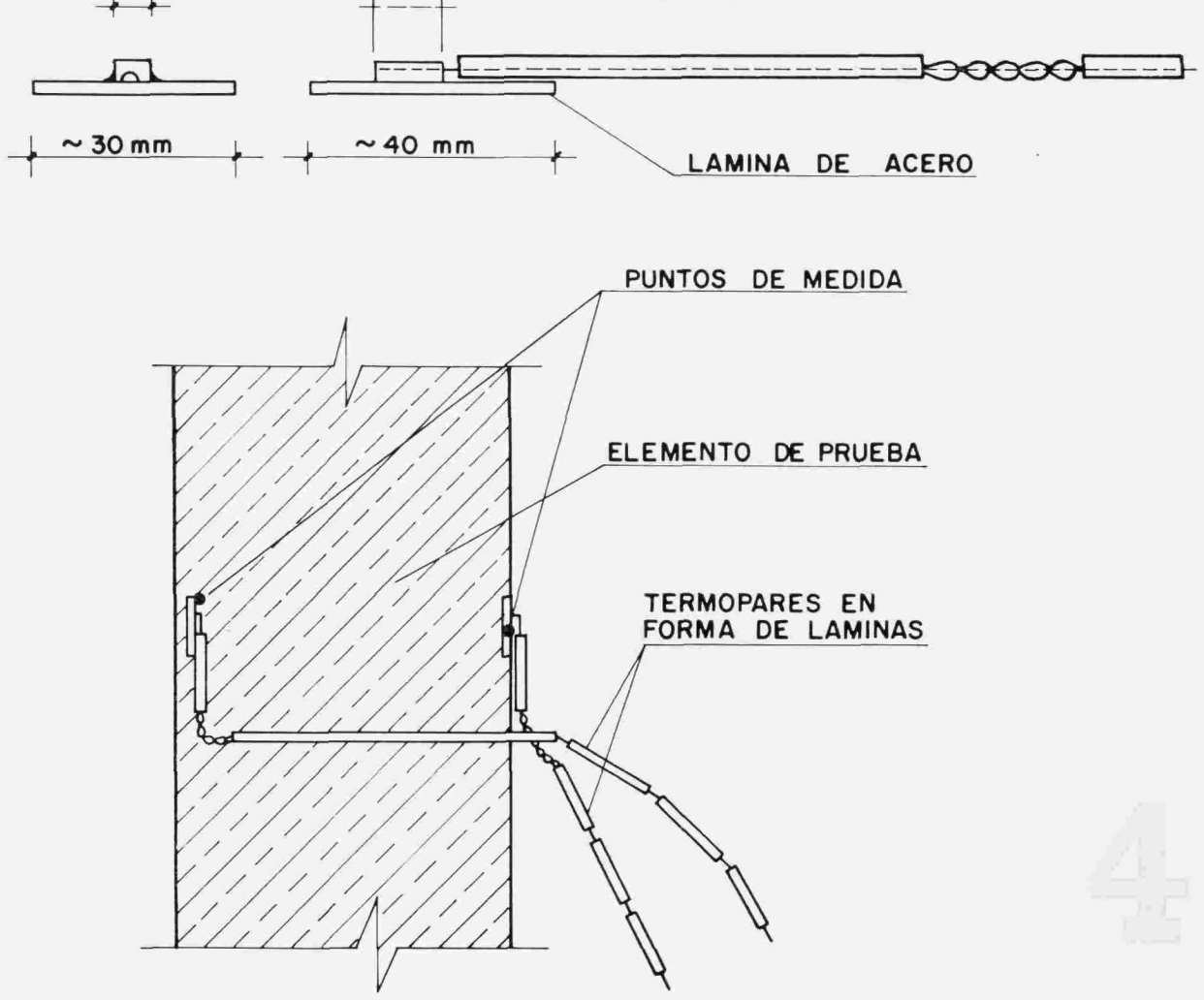
Los termopares empleados de cromo-aluminio, soldados y con refuerzos, resisten ampliamente temperaturas de $1.200^{\circ} \mathrm{C}$, y a corto plazo, temperaturas de $1.300^{\circ} \mathrm{C}$.

Para la medida de las temperaturas se han utilizado termopares de acuerdo a las temperaturas en la superficie calentada y no calentada de los elementos, así como en diferentes lu. gares característicos (fig. 5) de la sección, de tipo $a, b$, $c, d$ y $z$ termopares para la medida de las temperaturas de las barras de armadura de los forjados de tipo $w$ (soldados al acero durante la producción de los elementos), y termopares con protecciones de acero refractario "Marwedur» para la medida de la temperatura del local durante un incendio de tipo $m$.

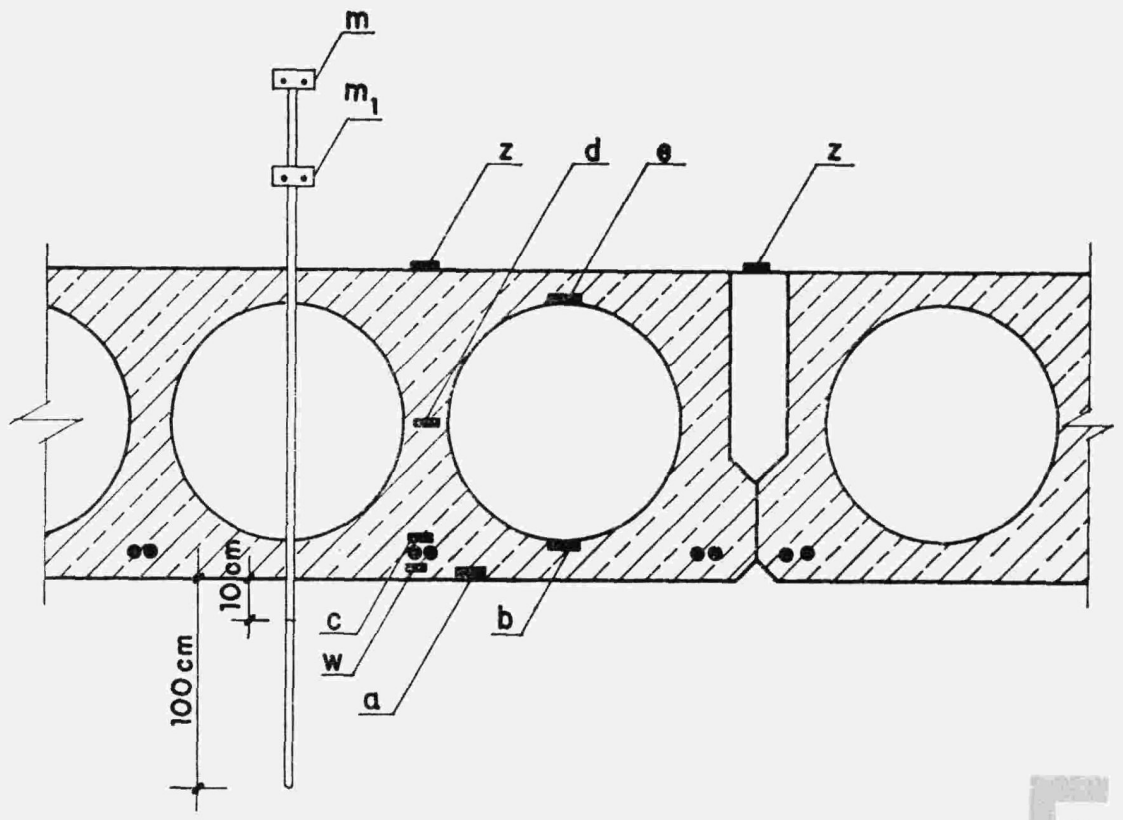

En los incendios se ha utilizado el mismo sistema de medida de la temperatura, con el empleo de los compensadores electrónicos automáticos y autográficos de 12 puntos. En el instrumento se puede observar directamente (fig. 6) la variación de las temperaturas en los puntos dados. Esto es particularmente importante en el caso del acero, donde se pudo observar el límite de elasticidad de este material.

En el programa de observaciones se prevé la investigación de la resistencia al fuego (desde 1 a 2 horas) de los elementos prefabricados de hormigón armado.

Mientras duró el incendio se realizaron observaciones permanentes del comportamiento de los bloques individuales, y de los elementos de la construcción; asimismo se anotaron los momentos característicos del incendio, tales como: la rotura y la caída de los vidrios; la caída del hormigón; el paso del humo y de las llamas a la planta superior y al exterior de las paredes; la influencia de los balcones; la influencia de las corrientes de aire; etcétera.

Si bien es cierto que los incendios a escala natural dependen de una serie de factores atmosféricos, tales como la intensidad del viento, la temperatura, la humedad, la presión, etc.; no obstante, en el caso de la (para la mayoría de los parámetros) curva normativa obtenida (en particular en la segunda fase del incendio, la más esencial, desde el punto de vista constructivo, debido a la posibilidad de la formación de las deformaciones duraderas en los elementos), es-

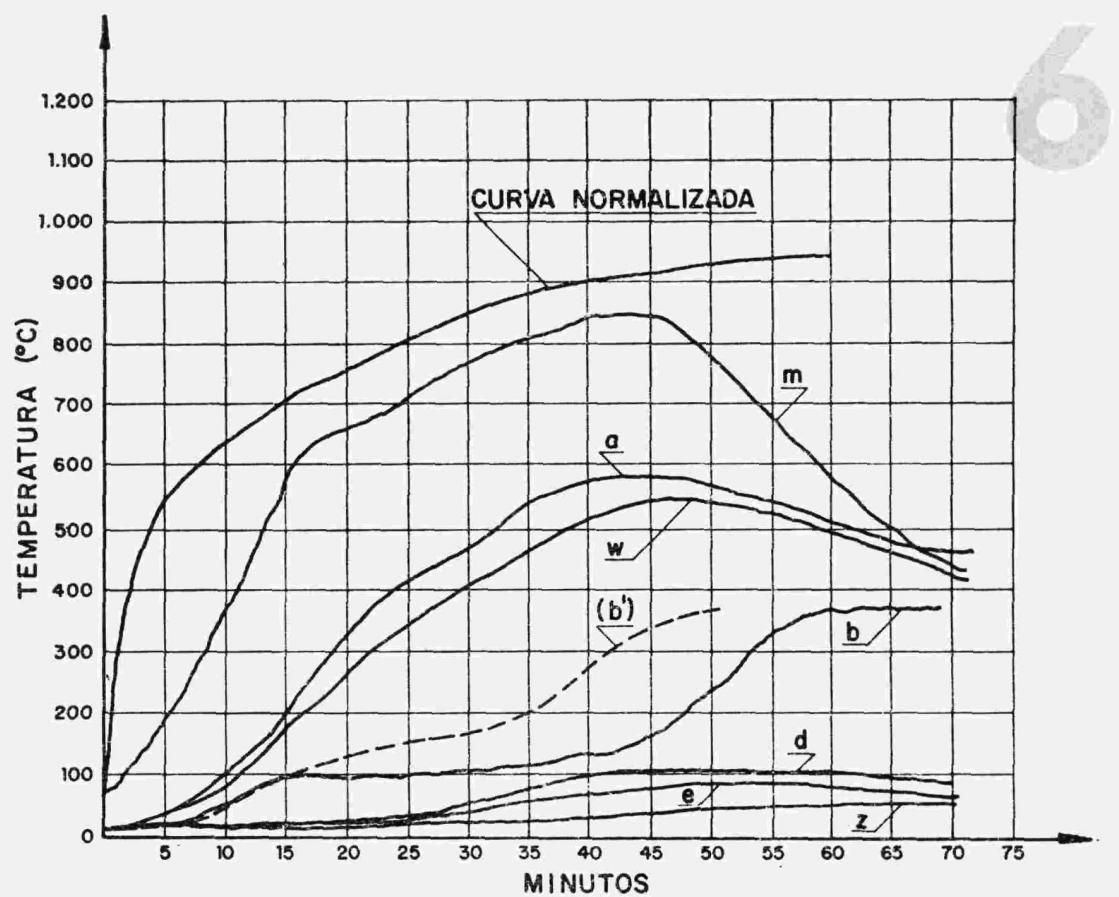


tas pruebas permiten contestar a una serie de preguntas esenciales pretensadas en la práctica normal de construcción y también resolver la protección contra incendios.

\section{Dtros métodos}

\section{Las pruebas a escala reducida}

Estas investigaciones pueden ser divididas en dos grupos: investigaciones a escala reducida, que se refieren a la influencia de las altas temperaturas sobre determinados materiales, y las investigaciones que tienen por objeto la determinación de las posibles relaciones existentes entre las investigaciones a escala reducida y las investigaciones más costosas a escala natural.

Valoración de la obra y de sus elementos según el sistema de puntos

Este método permitió, de un lado, precisar en forma muy estricta los rasgos de la obra y, de otro, dar la posibilidad de un enfoque lógico de las desviaciones de los principios comúnmente admitidos en el marco de la disminución de las clases de las resistencias al fuego en condiciones concretas. El sistema de puntos determina la resistencia al fuego, dependiendo de los rasgos materiales y constructivos normalmente admitidos para la determinación de la carga del fuego, de las zonas de incendio, la altura de la obra, de las vías de salida de emergencia, así como contemplar en tales momentos el estado de los extractores para la salida de humos y de calor, el nivel de las instalaciones de apagado y de señalización, así como la habilidad de los cuerpos de bomberos en función de la distancia, es decir, factores que tengan una gran influencia sobre el grado de la amenaza que gravita sobre las obras por causa del fuego.

\section{Caracterústica de las estructuras de hormigón ammado en condiciones de altas temperaturas}

El acero de armadura, bajo la influencia de altas temperaturas, rebaja sus cualidades fundamentales: la resistencia a la tracción $\mathbf{R}_{m}$, el límite de elasticidad $\mathbf{R}_{e}, \mathrm{y}$ el módulo de elasticidad $\mathbf{E}_{s}$, en forma no lineal.

Debido a lo anteriormente mencionado, la protección del acero en las armaduras, de la acción de las altas temperaturas, es particularmente importante.
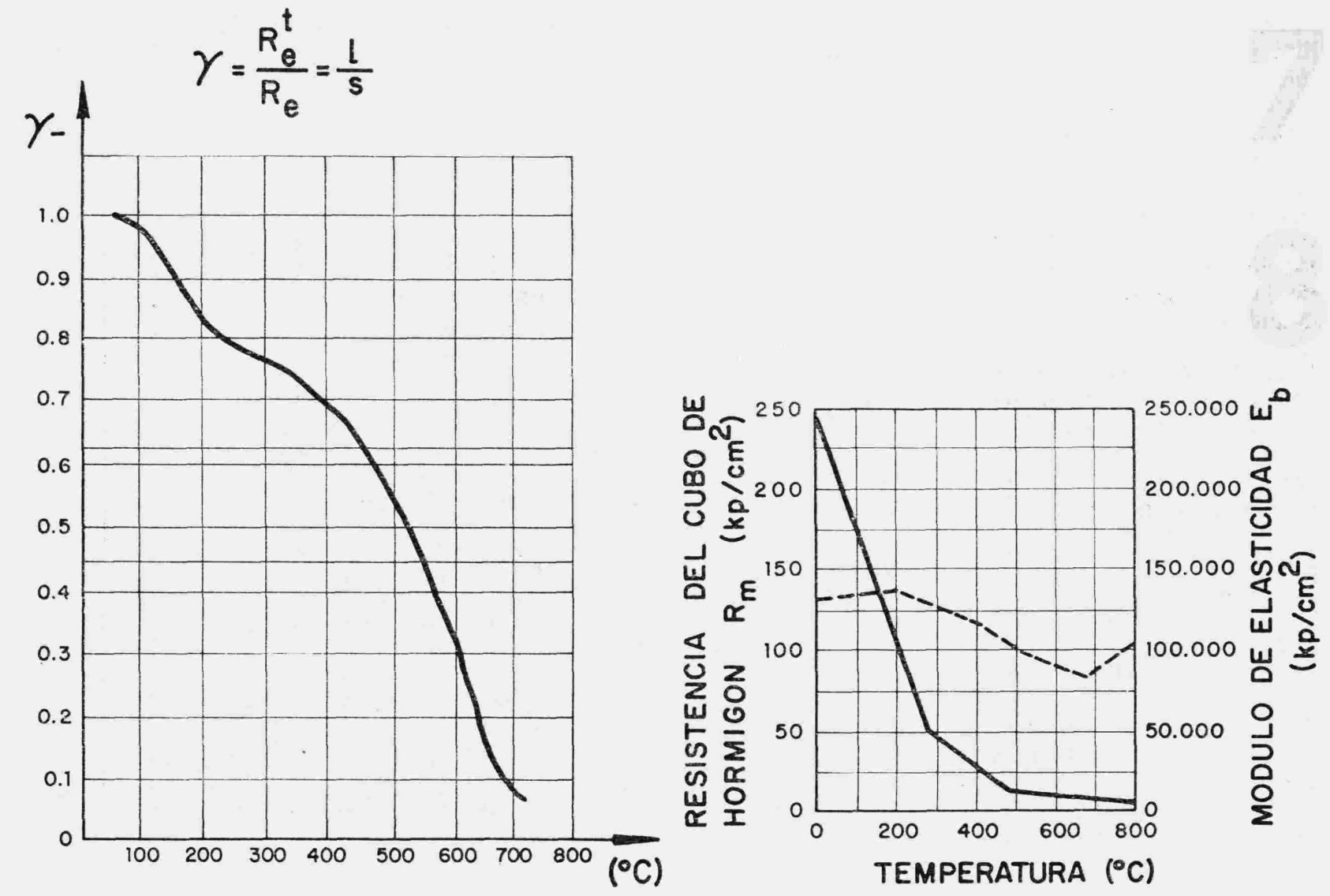
Las barras de las armaduras, cubiertas por una capa de hormigón del espesor correspondiente y sujetas a la acción de un fuego de poca duración, mediante la cual el acero no llegue a la temperatura de $400^{\circ} \mathrm{C}$, mantiene sus propiedades fundamentales, por lo que existen posibilidades de reparación de los forjados, pudiéndose evitar un cambio total, frecuentemente muy costoso.

La relación entre el límite de elasticidad $\mathbf{R}_{e}$ y la temperature para las clases de aceros soviéticos A-I, etc. y las categorías de acero st-0, st-3, st-5 nos la indica la figura 7. Observando esta figura resulta que hasta la temperatura de $400^{\circ} \mathrm{C}$ el acero de las armaduras mantiene sus propiedades fundamentales, y a temperaturas de $400^{\circ} \mathrm{C}$ el límite de elasticidad baja hasta $0,7 \mathbf{R}_{e}$; a temperaturas desde $400^{\circ} \mathrm{C}$ hasta $600^{\circ} \mathrm{C}$ el límite de elasticidad del acero disminuye considerablemente, y a temperaturas de $550^{\circ} \mathrm{C}$, hasta $0,5 \mathbf{R}_{e}$. Por encima de $600^{\circ} \mathrm{C}$ pierde totalmente su capacidad de transmisión de cargas.

El hormigón, bajo la influencia de altas temperaturas, disminuye su resistencia a la tracción $\mathbf{R}_{m}$, así como su módulo de elasticidad $\mathbf{E}_{b}$ (fig. 8). En la fase inicial de la acción con temperaturas de $100^{\circ}-130^{\circ} \mathrm{C}$ se elimina el agua químicamente libre. A partir de la temperatura de $350^{\circ} \mathrm{C}$ comienza la separación del agua químicamente unida.

Este proceso dura hasta la temperatura de $600^{\circ} \mathrm{C}$. A temperaturas de $500^{\circ} \mathrm{C}$ se produce la deshidratación del hidróxido de calcio. La descomposición del hidróxido de calcio en el mortero de cemento es la causa principal de la disminución de la resistencia del hormigón. A temperaturas próximas a $575^{\circ} \mathrm{C}$ se inicia la transformación de los cristales de cuarzo que se encuentran en el árido; ella contribuye también a la disminución de la resistencia del hormigón, aunque en una medida menor. A temperaturas de $600^{\circ} \mathrm{C}$ aumenta considerablemente el volumen del hormigón, lo que origina roturas en el elemento constructivo. Debido a que la estructura de las capas exteriores de hormigón, expuestas a la acción directa del fuego, está sujeta a la destrucción total, y la estructura de las capas, más alejadas de su superficie, sólo se halla expuesta a la destrucción parcial, existe la posibilidad de la eliminación de las destrucciones originales por la acción de las altas temperaturas sobre el hormigón. De esto resulta que la colocación correcta del acero de las armaduras en las estructuras de hormigón armado constituye un problema esencial. El acero -como se ha dicho antes - a la temperatura de $500^{\circ} \mathrm{C}$ pierde un $50 \%$ de su límite de elasticidad, previsto por las normas.

En el Instituto de Polonia habían sido realizadas las investigaciones de la consistencia al fuego de los elementos prefabricados ahuecados ́el espesor del elemento ahuecado es de $24 \mathrm{~cm}$; el espesor equivalente es de $12 \mathrm{~cm}$ ), de forjados de hormigón armado, y de paneles de forjados macizos de $9 \mathrm{~cm}$ de espesor.

Los resultados de estas investigaciones confirmaron que los ele-

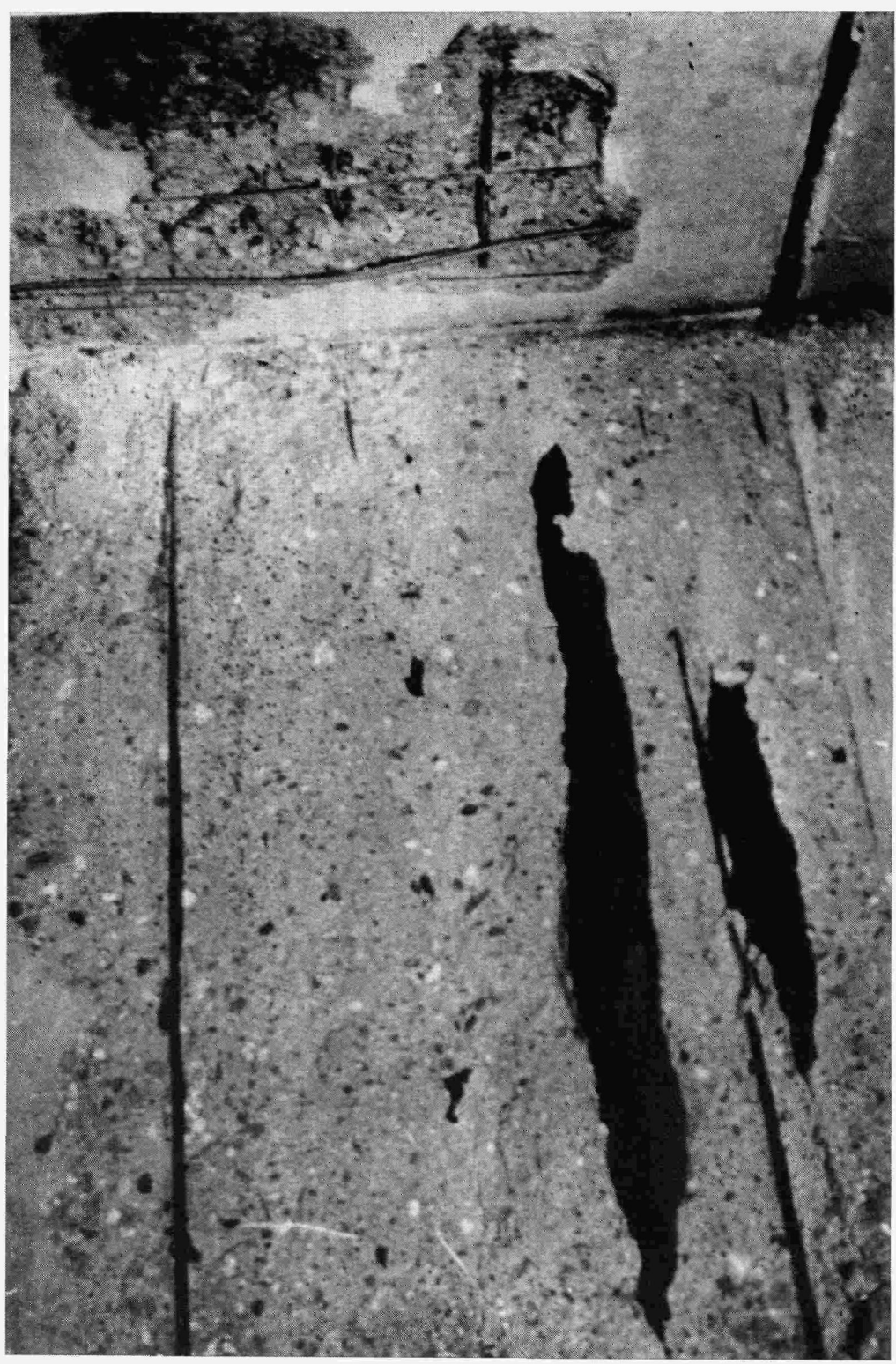


mentos ahuecados son más resistentes a la acción de las altas temperaturas que los elementos macizos.

Sobre la resistencia al fuego, fuera de los factores anteriormente mencionados, pueden tener influencia también: el peso del árido, el tipo de cemento, el tipo de hormigón y también los defectos en el proyecto y en la ejecución de los detalles estructurales. Por ejemplo, el empleo de los desperdicios del acero de armaduras como elementos (tacos) que garanticen el espesor correspondiente del recubrimiento en los elementos de forjados, empeora, en el caso de un incendio, la protección de la armadura principal; mientras que la ubicación del acero estructural (para montajes) en los elementos prefabricados de las paredes, demasiado cerca de sus superficies exteriores, puede acelerar, en el caso de un incendio, la caída superficial de las capas de hormigón (fig. 9).

\section{Dbservaciomes}

La revisión de la literatura, de las investigaciones y de las normas permite las siguientes constataciones:

Las pruebas al fuego en el horno de laboratorio, debido a las condiciones similares de las investigaciones realizadas (ISO, CAME), son las investigaciones más representativas, fácilmente comparables con los resultados de las investigaciones extranjeras.

Las pruebas contra el fuego, a escala natural, constituyen un complemento esencial de las investigaciones de laboratorio en el marco de la contemplación de diferentes influencias adicionales, difíciles de comprobar en el horno de laboratorio. Este tipo de prueba se recomienda para los elementos que han de ser producidos en gran escala.

Las pruebas contra el fuego, a escala reducida, permiten examinar los rasgos de los materiales, y las investigaciones de los modelos facilitan la programación y la determinación de las directrices de investigaciones más amplias.

El desarrollo actual de la prefabricación, careciendo de posibilidades para realizar investigaciones en los hornos de laboratorio, crea la necesidad de un estudio posterior más completo de los resultados extranjeros en este campo y determinar, con este punto de partida, las clases de resistencia al fuego para la mayoría de los elementos prefabricados utilizados en Polonia, así como la necesidad de editar un nuevo catálogo de tales elementos.

\section{Bibliografia}

Baranowski, L., y Ziolek, M.: «Odpornosć ogniowa wybranych elementów Żelbetonwych» (Resistencia al fuego de los elementos escogidos de hormigón armado empleados en la construcción industrializada). X Konferencia Naukowa Pzitb i Komitetu Inżynieri Ladowej Pan 1964.

Kochersheidt, W.: "Fuerwiderstandsfahigkeit von Beton und Stahlbeton». Technische. Hochschule, Stuttgart, 1963.

Ziolek, M.: «Characterystyka Konstrukcji Żelbetonych w Warunkach Wysokich Temperatur» (Característica de las estructuras de hormigón armado en las condiciones de altas temperaturas). Biuletyn-Informacja Oeiteb. Nr. 3, libr. III, 1968, Warszawa.

Ziolek, M.: «Metody Badan Odpornosci Ogniowej Elementów Konstrukcyjnych» (Métodos de las investigaciones de la resistencia al fuego de los elementos estructurales). Biuletyn-Informacja Oeiteb, Nr. 3, libr. VI, Warszawa, 1968.

Ziolek, M.: «Podstawowe Sposoby Zabezpieczania Konstrukcji Żelbetowich y Stalowych Przed Dzialaniem Wysokich Temperatur» (Métodos básicos de protección de las estructuras de hormigón armado y de acero de la influencia de altas temperaturas). Biuletyn - Informacja Oeiteb, Warszawa, Nr. 3, libr. V, 1968.

Normas polacas PN-64/B-02850, 02851, 02852.

Normas de U.R.S.S. SN-102-65.

Normas del CAME. Conferencia en Varsovia en el año 1965.

Normas inglesas 476: p. 2 y p. 3, 1953 у 1958.

Normas americanas ASTM E 69-50, E 102-58, E 119-58. 


\section{Résistance au feu des éléments structurumx puéfabriqués en bëtom anvéé}

Maciej Ziolek, ingénieur

Dans cet article l'auteur traite du comportement des structures et des éléments préfabriqués en béton armé face à l'action du feu, en vue d'adopter les épaisseurs de revêtements, les matériaux, les systèmes, les types de joints et même les conditions d'exploitation des édifices à bâtir, qui soient plus conformes à un minimum de propriétés déterminées d'isolement et de résistance.

\section{Strength mesistance of structural, prefobricuted, meinfonced concente elements}

Maciej Ziolek, engineer

This deals with the behaviour of structures and elements involving prefabricated reinforced concrete, when subjected to fire heat. Requirements are also given on adequate protection cover, materials, systems, and types of joints, as well as the correct use of buildings with these materials, so that the minimum necessary specifications on insulation and strength are met.

\section{Feuerfestigkeit von vorgefertigten Bauteilen ous Strolibetom}

Maciej Ziolek, Ingenieur

Dieser Artikel befasst sich mit der Feuerfestigkeit von Bauwerken und vorgefertigten Teilen aus Stahlbeton, im Hinblick auf die notwendige Dicke der Verkleidung, die Materialien, Systeme, Arten der Fugen und sogar die Nutzung der geplanten Bauten, um ihnen bessere und weniger aufwendige Isolier- und Festigkeitseigenschaften zu verleihen. 